\title{
Justiça climática e as estratégias de adaptação às mudanças climáticas no Brasil e em Portugal
} PEDRO HENRIQUE CAMPELLO TORRES,I ALBERTO MATENHAUER URBINATTI, ${ }^{I I}$ CARLA GOMES, III LUÍSA SCHMIDT, IV ANA LIA LEONEL, ${ }^{V}$ SANDRA MOMM, ${ }^{V I}$ e PEDRo Roberto JACOBI VII

\section{Introdução}

$\mathrm{E}$

STE ARTIGO busca contribuir para a ampliação do debate sobre justiça climática com problematização dessa questão a partir da literatura contemporânea e da revisão dos planos e estratégias nacionais de adaptação às mudanças climáticas no Brasil e em Portugal, como estudos de caso. A justiça climática e a equidade mundial são imperativos defendidos no Acordo de Paris, embora com singularidades, entendimentos e distinções em cada região do planeta (Kashwan et al. 2020). No entanto, há uma urgência por maior proatividade coletiva e por uma nova cultura cívico-ambiental.

Nesse sentido, torna-se imperativa a interface entre a agenda de direitos e justiça e o debate sobre as mudanças climáticas, mais especificamente em relação à adaptação, particularmente no planejamento de territórios para a variabilidade climática futura (Torres et al., 2021; Kashwan, 2017). Isso oferece a possibilidade de considerar o componente de justiça e redução das desigualdades na construção de políticas, projetos e planos de adaptação, sejam municipais, provinciais, sejam federais, regiões, bacias, empresas ou sociedade civil, mesmo como estratégia de planejamento da comunidade, insurgente e participativa.

Inicialmente restrita a discussões envolvendo pequenos países insulares que, como resultado da elevação do nível do mar, tendem a desaparecer, a agenda de adaptação cresceu em países como Holanda, Bangladesh e Dinamarca, entre outros. Mas como a agenda política e as estratégias de adaptação estão ocorrendo em diferentes realidades socioterritoriais, como no caso de Brasil e Portugal? Esse é o principal objetivo e contribuição para a agenda de pesquisa emergente em justiça climática, buscando elementos para fomentar um diálogo entre os dois países, com base em experiências no campo das políticas públicas climáticas. 


\section{Metodologia}

Foi realizada uma Revisão Sistemática de Literatura (RSL) sobre o tema da justiça climática em duas plataformas: Google Scholar e Web of Science. Junto com o descritor "Brasil" ou "Brazil" foram utilizados os descritores "justiça climática", "injustiça climática", "climate justice", "climate injustice". Junto a "Portugal" foram adotados os descritores "justiça climática", "injustiça climática", "climate justice" e "climate injustice".

Considerando que na literatura europeia a expressão "justiça climática" está tipicamente associada a artigos sobre negociações internacionais, relações Norte/Sul e países em desenvolvimento, ou seja, não captura a abrangência dos estudos sobre os impactos das alterações climáticas nas desigualdades sociais em nível nacional, decidimos recorrer a termos adicionais para a revisão de literatura sobre Portugal. Assim, consideramos adicionalmente as expressões "Portugal" e "alterações climáticas" associadas ao termo "justiça", assim como os descritores "energy poverty" ou "pobreza energética".

Foram encontrados 26 artigos para o caso brasileiro e 18 para o caso português. A seleção dos artigos se deu mediante critérios de inclusão e exclusão. O critério de inclusão considerou artigos científicos publicados em revistas nacionais e internacionais que tenham como foco principal a discussão da justiça ou injustiça climática no Brasil e em Portugal.

Além disso, foram considerados aqueles open access e os possíveis de serem acessados a partir da Universidade de São Paulo ou da Universidade de Lisboa. O critério de exclusão foi aplicado para que não fosse considerada "grey literature", tais como artigos publicados em anais de congressos, apresentação de trabalhos em congressos, working papers, livros e capítulos de livro. O tema da justiça climática aparece desde 2010 na literatura encontrada. Notou-se que existem alguns picos de publicação em ambos os países, como observados nos anos 2012, 2014, 2016 e 2019.

Posteriormente, analisamos o Plano Nacional de Adaptação (PNA) do Brasil e a Estratégia Nacional de Adaptação às Alterações Climáticas (Enaac) de Portugal e como as noções de justiça, vulnerabilidades e desigualdades são abordadas em cada um dos instrumentos de política. Para isso, foi realizada uma investigação em busca de descritores que refletissem a discussão do conceito de justiça em documentos oficiais de adaptação climática.

Para o caso brasileiro analisamos: os três volumes que compõem o PNA, a Revista Adaptação, produzida pelo Ministério do Meio Ambiente no processo de elaboração do plano, e a plataforma online AdaptaClima, que coleta e disponibiliza à sociedade conhecimento sobre adaptação climática. Nesses documentos, procuramos os descritores "justiça", "justa(s)", "justo(s)", "direito", "vulnerabilidade(s)", "meio ambiente / meio ambiente(ais)", e "desenvolvimento". 


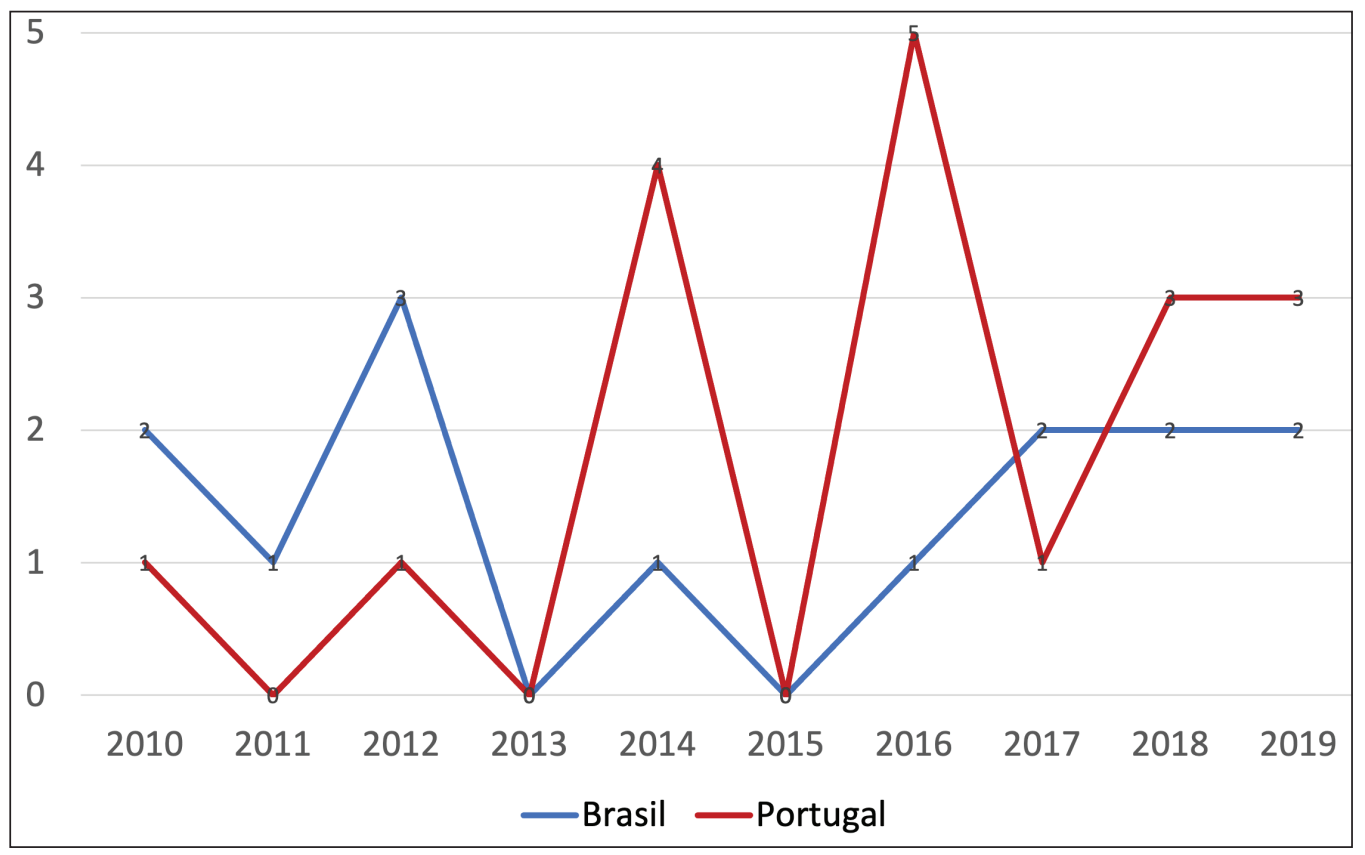

Figura 1 - Revisão Sistemática de Literatura sobre Justiça Climática. Fonte: Elaborada pelos autores.

No caso de Portugal, o foco da análise foram os principais instrumentos orientadores das políticas de adaptação em Portugal, nomeadamente a Estratégia Nacional de Adaptação às Alterações Climáticas, nas suas versões de 2015 e 2020, bem como os nove relatórios setoriais elaborados neste âmbito. Fizemos uma pesquisa sistemática dos descritores "justiça”, “justa(s)”, “justo(s)”, “direito", "vulnerabilidade(s)", "vulnerável(eis)" e "desigualdade(s)", todos estes associados ao termo "alterações climáticas".

\section{Justiça climática no Brasil e em Portugal}

\section{Brasil}

No contexto brasileiro, Zagonari (2010) discute justiça entre os critérios para a avaliação da água subterrânea no Brasil. $\mathrm{O}$ autor aplica a teoria chamada optimal-control em um contexto das mudanças climáticas. No critério justiça, os impactos das alterações climáticas não devem forçar os dois setores abordados pelo autor, o econômico e o social, a consumir menos água subterrânea do que as suas necessidades básicas estimadas. No entanto, esse estudo não desenvolve propriamente um conceito mais elaborado de justiça climática. Já Rammê (2012) aprofunda o conceito mostrando que a ética da justiça climática é a ética social no contexto da distribuição dos riscos climáticos globais. $\mathrm{O}$ autor apresenta algumas premissas para uma agenda política que englobe justiça climática, tais como: o imperativo ético do conceito, a urgência da consideração do risco para os seres humanos em situação de maior vulnerabilidade social, a redução dos combustíveis fósseis, suprapartidarismo e estratégias de longo prazo em ní- 
veis locais, protagonismo dos países desenvolvidos na mitigação com apoio da responsabilidade comum, mas diferenciada.

Milanez e Fonseca $(2010,2011$, 2012) apresentam três artigos publicados sobre o tema. Os três textos trazem argumentos similares, com a preocupação em revisar as principais publicações sobre o conceito na literatura mundial e associá-lo à percepção de eventos climáticos no Brasil, particularmente sobre as causas das cheias. Os autores sugerem três resultados benéficos a partir da incorporação do conceito. O primeiro seria a visibilidade da justiça climática nos inúmeros eventos e fóruns que o país já recebe e promove. O segundo é o aumento das possibilidades de expressão das reivindicações de grupos mais afetados pelo clima. O terceiro trata do fortalecimento de algumas políticas públicas ao associarem tragédias de eventos climáticos extremos como efeitos da própria mudança climática.

Ioris et al. (2014) mostram que os princípios da justiça climática deveriam orientar políticas pelo fato de serem a estratégia mais apropriada no enfrentamento da desigualdade e injustiça relacionadas às mudanças climáticas. Para isso, trazem um estudo de caso da região do Pantanal, que engloba Brasil, Paraguai e Bolívia. Os resultados evidenciam que a falta de estratégias de adaptação para a maioria da população compreender e lidar melhor com os efeitos do clima, bem como mecanismos participativos para a democratização do acesso aos recursos ambientais contribuem para a injustiça climática na região de estudo. A incorporação do conceito em políticas públicas, principalmente nas decisões associadas ao Pantanal e à Bacia do Alto Paraguai, forneceria possibilidades mais robustas de adaptação considerando as diferenças entre grupos populacionais.

Dalla-Nora e Sato (2019) também trazem um estudo de caso brasileiro à luz da justiça climática. Mais especificamente, o estudo busca compreender as diferentes percepções sobre as mudanças climáticas no território quilombola de Mata Cavalo, no cerrado do Centro-Oeste. Em linhas gerais, destacam como a escassez de água tem mudado alguns hábitos da população local e como os saberes locais acerca da alimentação, por exemplo, estão prestes a sumir ante práticas de monocultura na agricultura. Em contraponto ao cenário rural, cita-se aqui o trabalho de Henrique e Tschakert (2020), que estudaram o caso do projeto Parque Várzeas do Tietê em São Paulo. Os autores discutem a justiça climática no bojo de respostas adaptativas ao problema das inundações urbanas. Eles reforçam que é necessário ir além de inovações do ponto de vista técnico-gerencial para se aprofundar nas considerações sobre a justiça social e ambiental. Desse modo, sugerem a importância de um engajamento produtivo entre as comunidades locais e o governo em trajetórias adaptativas e resilientes que possam contestar assimetrias de poder e promover espaços inclusivos de tomada de decisão.

Verdan (2016) aborda o conceito de justiça ambiental, bem como o de injustiça ambiental, a partir do debate sobre o desenvolvimento econômico e a partir dos Direitos Humanos (Verdan, 2017). Os dois artigos evidenciam a 
tensão advinda do processo de industrialização entre o desenvolvimento econômico e o meio ambiente ecologicamente equilibrado, embate que acaba por agravar as injustiças ambientais e climáticas. Desse modo, o reconhecimento das injustiças climáticas no território brasileiro, assim como as fragilidades das populações mais necessitadas pela óptica dos direitos humanos e de fraternidade, precisa acontecer tanto por parte dos grandes empreendimentos econômicos poluidores quanto pelo poder público e movimentos sociais.

Oliveira $(2017$, p.94) questiona se "não seria a justiça climática almejada uma perspectiva que precisa justamente enfrentar a responsabilidade restritiva assumida pelos organismos internacionais?". O autor revisa o conceito de justiça dentro do debate local-global, a distinção entre justiça social e justiça global, e a crítica para uma justiça global cosmopolita. O autor destaca a complexidade do debate em nível internacional em torno da responsabilidade dos danos causados por uns e sofridos por outros, destacando injustiças nos acordos internacionais de combate às mudanças climáticas.

Ao revisarem o conceito de justiça ambiental, Branco e Armada (2018) associam esse conceito ao debate das mudanças climáticas. A própria construção histórica do conceito deve ser referência para práticas políticas mais democráticas, na medida em que movimentos sociais são importantes interlocutores para denúncias de desigualdades ambientais. Os autores argumentam ser necessário que, além da incorporação à risca das medidas alinhadas no Acordo de Paris, seja superado o discurso global do desenvolvimento sustentável, pois estaria alinhado aos setores econômicos que mais contribuem para as mudanças climáticas.

Em argumento semelhante, Patrick Bond (2012) mostra que a justiça climática é uma forma de romper com um discurso de desenvolvimento racialmente segregador e patriarcal. Nesse sentido, argumenta que somente a justiça climática enquanto movimento pode resolver as crises atuais no planeta, visto que agrega uma forte militância ambientalista e socioeconômica. No entanto, ela precisaria lidar com o desafio de se tornar uma rede mais inclusiva para a juventude e mais coerente com outras agendas, não deixando de estabelecer cada vez mais novas conexões. Finalmente, Miranda e Firpo Porto (2012) analisaram a Rio+20 e a Cúpula dos Povos, argumentando que, enquanto o primeiro evento teve o discurso baseado na ecoeficiência, na economia verde e na governança global, evidenciando interesses do mercado; o segundo promove novas formas de produção de conhecimentos com base na solidariedade e na justiça climática. Os autores entendem que a saúde coletiva é um eixo de discussão importante para abordar questões como a democracia, a ética, os direitos humanos e a justiça ambiental.

\section{Portugal}

No contexto português, destaca-se o trabalho de Schmidt et al. (2012), no qual são abordados os desafios da adaptação na zona costeira de Portugal. Os autores avaliaram a importância de aspectos críticos nesse contexto: o apoio fi- 
nanceiro ao futuro da região costeira e a justiça social, trazendo questões como a possível realocação e participação cívica efetiva nas decisões comuns para o litoral.

Schmidt et al. (2014) e O’Riordan et al. (2014) sublinham a importância da justiça social, considerando os diversos tipos de populações residentes da zona costeira e recorrendo ao modelo da governança adaptativa. Os planos em vigor para a gestão das zonas costeiras, por exemplo, há muito tinham evidenciado os conflitos de justiça social que algumas medidas tipicamente acarretam, como a retirada de construções e deslocação de populações em zonas de risco. À época da publicação desses dois artigos, Portugal vivia um período de forte recessão econômica, e a redução do gasto público ameaçava agravar os problemas de justiça social, ao afetar diretamente os serviços, a segurança e o bem-estar da população litorânea portuguesa.

Fonseca (2014) reforça a importância da justiça climática dentro de uma perspectiva de discussão das relações internacionais de acordos globais sobre o clima. $\mathrm{O}$ autor cita a diferença entre justiça distributiva, no caso dos países mais desenvolvidos a assumirem a dívida histórica em relação às suas emissões, e a justiça corretiva, que engloba a consideração de que os países menos desenvolvidos sofrerão as piores consequências. O estudo mostra que há uma inconsistência temporal em relação ao combate às alterações no clima, pois, ainda que todos os cenários indiquem catástrofes futuras, não são considerados como cruciais os efeitos em longo prazo nas decisões globais. Em argumento semelhante, Colen (2014) publicou um comentário ao texto "Nações, gerações e justiça climática", de Axel Gosseries (2014), no qual aborda a questão da justiça por meio do seu caráter intergeracional. No entanto, o autor destaca inúmeras complexidades relativas a questões morais sobre a comunicação entre gerações não só em relação ao clima, mas também à cultura, política, educação, entre outros.

Sareen e Haarstad (2018) escreveram sobre três breves estudos de casos de absorção solar multiescalar em Portugal: campo de teste solar I na ecovila de Tamera, a plataforma de demonstração solar da Associação Enercoutim e o parque solar Amareleja. Foram comparados esses casos a partir de múltiplas espacialidades e questões normativas entre instituições, materialidade e relacionalidade relevante para as transições para energias sustentáveis. Os autores abordam o conceito de justiça energética dentro do cenário das mudanças climáticas, oferecendo uma abordagem analítica que pode servir como um facilitador para uma aprendizagem reflexiva que incorpore aspectos sociotécnicos e de justiça energética das transições sustentáveis.

Também Delicado et al. (2016) abordaram o investimento em energias renováveis em Portugal desde a perspectiva da justiça. Estudaram as comunidades vizinhas de três parques eólicos e de uma central solar, para concluir que há necessidade de melhor transparência dos processos de instalação destas unidades, bem como a sua justiça distributiva, assegurando que os benefícios econômicos cheguem às comunidades locais, que são também as mais atingidas pelos im- 
pactos negativos das instalações. Guerra e Schmidt (2016) analisaram que os Objetivos de Desenvolvimento Sustentável (ODS) são uma potencialidade para passar do whishfull thinking para integração efetiva de diferentes dimensões da sustentabilidade.

Viegas et al. (2014) publicaram artigo baseado em teorias compreensivas para analisar os sistemas climáticos, ambientais e socioculturais acerca do que eles chamaram de "racionalidades leigas", isto é, fontes de saberes plurais. Os autores citam Boaventura de Sousa Santos (2005) ao tratarem da "justiça cognitiva", dentro de uma busca por justiça social global. Segundo os autores, o processo de reflexão sobre a produção do conhecimento leigo "contribuirá para compreendermos como as pessoas percecionam e vivem com as mudanças climáticas e como lidam com os seus efeitos" (Viegas et al., 2014, p.359).

Otto et al. (2019) estudaram a influência dos chamados "Cursos Abertos Online" (MOOC em inglês) no aprendizado sobre as mudanças climáticas. Eles afirmam que não é possível uma compreensão real dessas alterações e das políticas climáticas sem a discussão da justiça climática, englobando as questões de justiça inter- e intrageracional, além dos aspectos morais da escolha de determinados instrumentos políticos. Foram avaliados dois estudos de caso, um na Alemanha e outro em Portugal. Em ambos os casos, a justiça climática foi o eixo comum e transversal, elucidando que a consideração da justiça é essencial independentemente da área de estudo.

Em paralelo, surgem nos últimos anos estudos que refletem sobre os aspectos de justiça social e de vulnerabilidade que se tornam ainda mais críticos num contexto de alterações climáticas. É o caso dos estudos sobre pobreza energética (Simões et al., 2016; Bartiaux et al., 2016, 2018; Horta et al., 2019; Gouveia et al., 2019), que evidenciam os elevados índices de vulnerabilidade social e econômica que levam a que uma elevada percentagem da população tenha dificuldade em aquecer ou arrefecer as suas habitações, em comparação com outros países europeus.

Nunes (2018) faz uma análise comparativa dos impactos desiguais das condições climáticas, em particular do frio, entre os idosos do Reino Unido e de Portugal, reforçando as condições de particular vulnerabilidade da sociedade portuguesa no contexto europeu. A pobreza energética e os impactos da temperatura na saúde dos grupos mais vulneráveis têm merecido maior atenção, mas há também estudos sobre as desigualdades entre as capacidades de adaptação das populações do país, que comparam os municípios do interior com os do litoral (Campos et al., 2017). Arsenio et al. (2016) centram-se num aspecto que tem sido menos explorado no contexto português, ao analisarem o impacto das políticas de mobilidade sustentável, associado à mitigação das alterações climáticas, nas problemáticas de equidade social.

As problemáticas da justiça processual em face das alterações climáticas, em particular como é que as percepções de justiça têm sido manifestadas pelo 
público e pela mídia, tem merecido também a análise dos cientistas sociais em Portugal, como é o caso de Carvalho (2010).

Tabela 1 - Quadro geral dos temas da justiça climática no Brasil e em Portugal

\begin{tabular}{|l|l|}
\hline Perspectivas & Autores \\
\hline Gestão de recursos & Zagonari (2010) \\
\hline Transições energéticas & $\begin{array}{l}\text { Simões et al. (2016); Delicado et al. (2016); Bartiaux et al. } \\
\text { (2016); Arsenio et al. (2016); Nunes (2018); Sareen e Haarstad (2018); } \\
\text { Horta et al. (2019); Gouveia et al. (2019) }\end{array}$ \\
\hline Político-institucionais e legislativas & $\begin{array}{l}\text { Rammê (2012); Schmidt et al. (2012); Schmidt et al. (2014); } \\
\text { loris et al. (2014); O’Riordan et al. (2014) }\end{array}$ \\
\hline Éticas e jurídicas & Verdan (2016, 2017); Fonseca (2014); Colen (2014) \\
\hline Percepções e discursos & $\begin{array}{l}\text { Milanez e Fonseca (2010, 2011, 2012); Carvalho (2010); } \\
\text { Viegas et al. (2014) }\end{array}$ \\
\hline Relações internacionais; & $\begin{array}{l}\text { Miranda e Firpo Porto (2012); Bond (2012); Guerra e Schmidt (2016); Oliveira } \\
\text { (2017); Branco e Armada (2018) }\end{array}$ \\
\hline Movimentos sociais e comunidades & Campos et al. (2017); Dalla-Nora e Sato (2019); Henrique e Tschakert (2020) \\
\hline Conhecimento e educação & Otto et al. (2019) \\
\hline
\end{tabular}

Fonte: Elaborada pelos autores.

\section{Justiça e desigualdades nas estratégias nacionais de adaptação às mudanças climáticas}

\section{Brasil}

O Plano Nacional de Adaptação (PNA) brasileiro foi elaborado por meio de um Grupo de Trabalho (GT) do Comitê Interministerial de Mudança do Clima (CIM), instituído pelo Decreto n.6.263/2007 de 21 de novembro de 2007. O GT era formado por membros de doze ministérios e demais agências e fundações, ou seja, com um caráter intersetorial, contando com documentos técnicos de especialistas para analisar as minutas do PNA e incorporar as sugestões provenientes da Consulta Pública de 2015.

Além da consulta pública, o Ministério do Meio Ambiente (MMA) brasileiro também realizou chamada pública para "coleta de subsídio" sobre temas pertinentes à construção do PNA e que o GT julgou que necessitava mais informações. Nessa coleta, foi disponibilizado por um mês, no final de 2014, no site do MMA, um formulário para contribuições.

Segundo o compilado das contribuições, chamou atenção a questão dos riscos climáticos, por setores e biomas, e foi sugerida a construção integrada do PNA. Muitos proponentes indicaram o PNA como "elemento de planejamento, disponibilizando informações e diretrizes que permitam promover o desenvolvimento econômico-social aliado às medidas necessárias para conservação dos recursos ambientais e redução da vulnerabilidade" (MMA, 2014, p.11), se aproximando de medidas e iniciativas locais de adaptação e como "elemento indutor 
de uma estratégia de articulação federativa" (ibidem) harmonizando com políticas climáticas existentes entre os entes subnacionais.

Buscando pelos descritores investigados, "desigual" não apresentou nenhuma citação, enquanto "just”" (raiz das palavras justa, justiça e derivações) retornou apenas uma, na resposta do Observatório do Clima para a questão sobre o que é necessário para se adaptar a mudança do clima. Dentre os demais insumos recebidos e disponibilizados no site do MMA, apenas aquele intitulado "Contribuição da Fundação Boticário sobre Adaptação Baseada em Ecossistemas" (AbE), elaborado em parceria com o Iclei (Governos Locais pela Sustentabilidade), introduz no debate a questão da justiça, quando apresenta princípios e escopo de projetos de $\mathrm{AbE}$, sendo um dos princípios a Equidade e a promoção da justiça.

Também foi realizada uma consulta pública, no final de 2015, e cinco oficinas regionais sobre o PNA. Fazendo a mesma busca nos documentos relacionados à consulta, o relatório não apresenta citação "justo" ou "desigual", enquanto o compilado de respostas à consulta, apesar de não abordar os termos, apresenta repetidas vezes preocupações em torno da desigualdade social e ambiental relacionada à intensificação da vulnerabilidade de setores sociais diante das mudanças climáticas. Nas oficinas, o termo justiça é mencionado no encontro de Fortaleza, na qual se menciona que "A Justiça Climática deve ser o princípio norteador do ônus e bônus das demandas necessárias à redução da vulnerabilidade".

Embora o PNA mencione a população mais vulnerável, o descritor "justiça" não aparece no sumário executivo ou no volume 1 - estratégia geral. Neste segundo, o descritor "justo" aparece entre os princípios, que se refere à

[...] adaptação como estratégia para promover o setor produtivo com uma transição justa para trabalhadores e crescimento econômico, alinhada às estratégias de redução da pobreza, desigualdades socioeconômica e regional, incluindo a consideração do princípio de prevenção e precaução. (Brasil, 2016b, p.19)

O segundo volume do PNA para as mudanças climáticas trata de estratégias setoriais e temáticas e, em seu escopo, utiliza o descritor "justiça” quatro vezes, uma delas para explicar o escopo de outro plano (Plano Nacional de Promoção de Produtos da Sociobiodiversidade), articulado ao PNA como guia para promover a adaptação por meio da estratégia de Segurança Alimentar e Nutricional (Brasil, 2016c, p.233). No terceiro volume do plano (Brasil, 2016c), intitulado Estratégia da Cidade, o descritor "justiça" está vinculado ao debate que pontua que, embora todos sejam afetados de alguma forma pelas mudanças climáticas, os impactos são mais graves para os mais pobres.

Nesse sentido, o plano também é apresentado como um instrumento de articulação intersetorial e intergovernamental no desenvolvimento da capacidade de resposta. No capítulo sobre a estratégia de aldeias e populações vulneráveis, 
especificamente na seção Povos Indígenas e Mudanças Climáticas: Vulnerabilidade, Adaptação e Conhecimento Tradicional, há uma menção ao descritor "Justiça Climática" (Brasil, 2016c, p.159). A ideia desse descritor é que aqueles que são menos responsáveis pelos impactos que levam às mudanças climáticas, como as emissões de gases de efeito estufa, são os mais afetados pelos efeitos das mudanças climáticas.

A principal estratégia apresentada é baseada na "inserção da gestão de riscos das mudanças climáticas nas políticas e planos públicos setoriais e temáticos existentes, bem como nas estratégias nacionais de desenvolvimento" (Brasil, 2016a p.5). Esse documento reconhece a vulnerabilidade das populações tradicionais (indígenas, quilombolas, ribeirinhos) ao risco associado às mudanças climáticas e que deve haver abordagens com critérios raciais, étnicos e de gênero (Brasil, 2016b, p.19). Entre as estratégias setoriais apresentadas pelo plano, algumas referem-se a povos e populações vulneráveis, com o objetivo de promover sua adaptação por meio de três objetivos (Brasil, 2016a, p.11): a) "Diagnóstico de vulnerabilidade à mudança climática das populações atendidas pela Política Nacional de Gestão Territorial e Ambiental das Terras Indígenas (PNGATI)”; b) Diagnóstico da vulnerabilidade às mudanças climáticas das populações atendidas pelo Plano Nacional de Segurança Alimentar e Nutricional (Plansan); c) Diagnóstico preparado e redução da vulnerabilidade às mudanças climáticas promovidos em populações vulneráveis e beneficiárias de políticas públicas agro-extrativas.

Além do próprio plano, a Secretaria de Mudanças Climáticas e Qualidade Ambiental do Ministério do Meio Ambiente produziu a Revista Adaptação (Brasil, 2016d), durante consultas públicas para a construção do PNA, voltadas para técnicos, sociedade civil, empresários e pesquisadores. O objetivo da revista é a busca pelo diálogo a partir das diferentes opiniões dos parceiros envolvidos no processo de elaboração do PNA. Os descritores "justiça”, "justo(s)", "justa (s)" ou "direito" não aparecem em nenhuma vez. Podemos dizer que isso não se deve à falta de dados, indicadores ou resultados científicos, pois, como discutido aqui, a relação entre vulnerabilidade social e ambiental é crucial para políticas relacionadas à adaptação às mudanças climáticas.

Na biblioteca de conteúdo da plataforma AdaptaClima, coordenada pelo Ministério do Meio Ambiente do Governo Federal, não há uma única menção à palavra justiça na extensa lista de conteúdos. A plataforma foi lançada em 2017 para alcançar o primeiro objetivo do PNA, que inclui uma plataforma on-line para gestão do conhecimento em adaptação e disponível para a sociedade.

A noção de "justiça climática" não possui centralidade no Plano Nacional de Adaptação (Torres et al., 2020). Mas, como pode ser visto pelo levantamento da literatura sobre o descritor, o tema não foi incorporado na agenda pública, seja do governo, dos movimentos sociais, das organizações não governamentais ou academia. Em um país que historicamente priorizou ações de mitigação, sobretudo em relação às queimadas na Amazônia - principal vetor de emissão de gases 
de efeito estufa - o tema da Adaptação ainda é bem incipiente. Resultado disso é o pequeno número de planos e política de Adaptação, seja em âmbito nacional, federal ou estadual, mesmo após o Acordo de Paris (Espindola; Ribeiro, 2020). A tendência, por outro lado, é de aumento exponencial tanto de planos de adaptação, quanto da agenda de justiça climática no país. Trata-se, nesse sentido, de um janela de oportunidades para que esses novos planos climáticos incorporem desde a concepção questões centrais relacionadas à desigualdades, justiça e direitos. Do contrário serão apenas narrativas capturadas pelo por governos, ONG ou grupos de elites, vendendo falsas soluções ou soluções que perpetuam a enorme desigualdade existente no país.

\section{Portugal}

O quadro político-institucional sobre as alterações climáticas em Portugal iniciou-se na sequência da ratificação da Convenção-Quadro das Nações Unidas sobre as Alterações Climáticas em 1992 e, especialmente, com a assinatura do Protocolo de Quioto em 1998 (ratificado em 2002), quando foi criada a Comissão para as Alterações Climáticas (CAC), uma estrutura interministerial composta por funcionários de vários ministérios, sem representantes da sociedade civil. Essa Comissão foi responsável pela elaboração de uma estratégia nacional para as alterações climáticas e pelo acompanhamento, a nível nacional e internacional, do processo de tomada de decisões e da implementação de políticas.

As políticas públicas sobre alterações climáticas começaram, contudo, a adquirir relevância apenas em meados da década de 2000 (Schmidt; Delicado, 2014). O Programa Nacional para as Alterações Climáticas (Pnac) foi aprovado em 2004 com medidas para múltiplos setores como a produção de eletricidade (com meta de 39\% de eletricidade gerada de fontes renováveis até 2015), eficiência energética em edifícios, transportes públicos e privados, agricultura e florestas. Pouco mais tarde, as "Novas Metas 2007" vinham reforçar estas medidas, colocando ênfase no aumento da produção de eletricidade por fontes renováveis e na eficiência energética de novos edifícios.

O quadro de políticas de resposta às alterações climáticas em Portugal centrou-se, portanto, numa primeira fase, seguindo as tendências europeias e globais, em ações de mitigação. O passo decisivo no arranque de uma política de adaptação, perante a evidência de que alguns impactos das alterações climáticas já eram inevitáveis, deu-se com a aprovação da Estratégia Nacional de Adaptação às Alterações Climáticas (Enaac) em 2010, seguindo uma diretiva da União Europeia (UE) (a Estratégia da UE apenas foi aprovada em 2013).

Considerou-se importante, portanto, abranger esses documentos na análise. No entanto, apenas no relatório setorial dedicado à Saúde Humana se reflete em maior detalhe sobre os grupos sociais mais vulneráveis às alterações climáticas, referindo-se especificamente aos efeitos das ondas de calor (sobretudo a de 2003) entre as populações mais idosas e os portadores de patologias 
cardiovasculares ou respiratórias, em particular quando estes fatores se conjugam com uma posição econômica desfavorecida.

O ano de 2015, após a crise econômica e sob um novo governo de centro-esquerda, foi um marco no campo da política nacional de alterações climáticas, com a aprovação do Quadro Estratégico para a Política Climática que incluiu um novo Programa Nacional para as Alterações Climáticas (Pnac 2020-2030), uma nova Estratégia Nacional de Adaptação às Alterações Climáticas (Enaac 2020) que retoma os mesmos setores de atuação e a criação da Comissão Interministerial do Ar e das Alterações Climáticas (Ciaac) que, além dos representantes dos ministérios, dessa vez integra cientistas de várias áreas e também representantes associativos.

Em 2016, Portugal ratificou o Acordo de Paris, sob a promessa feita pelo primeiro-ministro na COP 22 de que o país seria "neutro em carbono" em 2050, e em 2018 foi apresentado à consulta pública o Programa de Ação para a Adaptação às Alterações Climáticas (P-3AC). Esse programa complementa e sistematiza os trabalhos realizados no contexto da Enaac 2020 tendo em vista o seu segundo objetivo - a implementação de medidas de adaptação, elegendo oito linhas de ação concretas de intervenção direta no território e nas infraestruturas.

Estas linhas de ação são complementadas por outra linha de caráter transversal, para responder aos principais impactos e vulnerabilidades identificados para Portugal, e que inclui o desenvolvimento de ferramentas de suporte à decisão, tal como ações de capacitação e de sensibilização.

Na transição de 2018 para 2019 surgiram dois novos planos estratégicos. O Plano Nacional Integrado de Energia e Clima (Pnec) e o Roteiro para a Neutralidade Carbônica 2050, que tem o objetivo geral de suportar tecnicamente o compromisso assumido por Portugal de atingir a neutralidade carbônica da sua economia até 2050, com trajetórias específicas de transição nos setores da energia, mobilidade e transportes, indústria, setor residencial e de serviços, agricultura e florestas, resíduos, incluindo as transições induzidas pela economia circular.

Ao longo da última década, foi nítida uma preocupação crescente com o alargamento dos processos de consulta pública destes planos e, por conseguinte, com a sua justiça processual. A elaboração do RN2050 foi acompanhada de uma sucessão de debates públicos temáticos, que se estenderam ao longo de mais de um ano. Um dos grupos de trabalho criados no âmbito deste processo centrou-se precisamente na "Transição Justa", com o objetivo de minimizar o impacto social das medidas de descarbonização, em particular no emprego, devido ao encerramento de unidades industriais, e na alteração dos padrões de mobilidade. Contudo, nem sempre se traduzem efetivamente numa maior diversidade de vozes, ou asseguram que essas vozes têm efetivamente influência nas decisões que acabam por ser tomadas.

Ao analisarmos os instrumentos de política especificamente centrados na adaptação, verificamos de fato que a justiça apenas surge como um tema explícito 
e uma prioridade muito recentemente. Nem na Estratégia Nacional de Adaptação, publicada em 2010, nem mesmo na sua versão mais recente, revista em 2015 (Enaac 2020), se encontram ocorrências do termo. A única que pudemos identificar é a referência aos usos do solo no contexto da fiscalidade verde que, segundo o documento, deve contribuir para promover "uma utilização justa e sustentável do solo, do território e do espaço urbano e introduzindo sinais que facilitem a transição tendencial para uma economia de baixo carbono".

Uma pesquisa por termos relacionados com o conceito de justiça, como vulnerabilidade, evidencia que apenas têm ocorrência num contexto genérico e referem-se, na maior parte das vezes, às vulnerabilidades físicas do país. As pesquisas pelos descritores "direito" ou "desigualdade (s)" retornam também resultados nulos em ambas as Enaac, uma vez que, entre as poucas ocorrências, nenhuma está relacionada com questões de justiça ou de vulnerabilidade social.

Essa análise evidencia que a justiça não surge como uma preocupação explícita nos principais instrumentos orientadores da política de adaptação em Portugal, mesmo quando considerados conceitos relacionados como as desigualdades e vulnerabilidades sociais.

O fato de o tema da justiça aparecer de forma explícita no Roteiro da Descarbonização (2019), contemporâneo destes planos - sob a expressão "Transição Justa" - confirma e reforça a conclusão de que a justiça (seja referida como social, ambiental ou climática) tem uma aparição muito tardia nas políticas climáticas em Portugal. Em suma, a análise das estratégias de adaptação às alterações climáticas em Portugal revela que as preocupações com a justiça, as desigualdades e as vulnerabilidades sociais apenas foram absorvidas pelo discurso político, e pelos instrumentos de política, depois dos movimentos sociais as terem trazido à esfera pública. Apenas em 2018 e 2019 a justiça surge como tema explícito nas políticas sobre as alterações climáticas em Portugal.

\section{Conclusões preliminares}

Enquanto persistirem as desigualdades no Brasil, em Portugal e em outros territórios do planeta, o chamado à ação contra as mudanças climáticas será, sobretudo, contra as desigualdades ambientais. A adaptação e a justiça devem estar tão entrelaçadas no planejamento, quanto nas políticas e na mobilização social, para que a adaptação e a justiça climática não se tornem simplesmente conceitos vazios ou reprodutivos da produção de desigualdades nos territórios.

A pesquisa empírica na revisão da literatura evidenciou que o conceito de justiça climática tem sido utilizado no contexto das negociações internacionais e não tanto para estratégias de políticas públicas nacionais. No caso de Portugal, a justiça enquanto princípio surge primeiro como "bandeira" de movimentos sociais, e apenas muito recentemente é transposta para os planos de ação climática.

No caso do Brasil a expressão "justiça climática" ainda está distante da agenda política e de pesquisa, embora seja possível observar um movimento influenciado pela agenda de mobilizações internacionais ou por grandes organiza- 
ções não governamentais. Trata-se de tema que merece ser explorado em pesquisas futuras, com foco sobre porque movimentos de base, partidos e organizações sociais brasileiras não assumiram a agenda da Justiça Climática, que, de alguma maneira, poderia integrar questões relacionadas ao estudo dos impactos das mudanças climáticas e a redução das desigualdades em nosso país.

O fato que parece claro é que não há definição única de justiça climática, menos ainda de sua tradução ou captura para agenda de governos ou movimentos. Nesse sentido, perceber as diferenças entre os "Movimentos de Ação Climática" que não devem ser entendidos como sinônimos de "Movimentos por Justiça Climática" como sugere Kashwan (2021), é crucial para um olhar crítico sobre a trajetória dessas noções e suas possibilidades como práxis realmente transformadora face aos desafios planetários contemporâneos.

Agradecimentos - Agradecemos à Fundação de Amparo à Pesquisa do Estado de São Paulo (Fapesp), processos n. 2018/06685-9, 2019/05644-0, 2019/184627, 2015/03804-0, 2016/25375-5 e 2018/02464-8, 2018/12245-1.

\section{Referências}

AGÊNCIA PORTUGUESA DO AMBIENTE (APA), ENAAC - Estratégia Nacional de Adaptação às Alterações Climáticas, Resolução do Conselho de Ministros de Portugal n.24/2010, de 18 de março.

. ENAAC - Estratégia Nacional de Adaptação às Alterações Climáticas: Relatórios Setoriais, 2012.

ENAAC 2020 - Estratégia Nacional de Adaptação às Alterações Climáticas, Resolução do Conselho de Ministros de Portugal n.56/2015, de 30 de julho.

ARSENIO, E.; MARTENS, K.; DI CIOMMO, F. Sustainable urban mobility plans: Bridging climate change and equity targets? Research in Transportation Economics, v.55, p.30-9, jun. 2016.

BARTIAUX, F. et al. Social diffusion of energy-related practices and representations: Patterns and policies in Portugal and Belgium. Energy Policy, v.88, p.413-21, 2016.

BARTIAUX, F. et al. Energy justice, unequal access to affordable warmth, and capability deprivation: A quantitative analysis for Belgium. Applied Energy, v.225, p.1219-33, 2018.

BOND, P. Crescimento verde inclusivo do Banco Mundial, Rio+20 e BRICS como ameaças à justiça climática. Tensões Mundiais, v.8, n.15, p.93-133, 2012.

BRANCO, M. G.; ARMADA, C. A. S. A. Desafios e perspectivas para a justiça ambiental face à nova realidade das mudanças climáticas. REBELA - Revista Brasileira de Estudos Latino-Americanos, v.8, n.2, 2018.

BRASIL. Ministério do Meio Ambiente. Plano Nacional de Adaptação à Mudança do Clima: sumário executivo. Brasília, DF: MMA, 2016a. 
BRASIL. Ministério do Meio Ambiente. Ministério do Meio Ambiente. Plano Nacional de Adaptação à Mudança do Clima: volume 1 - estratégia geral. Brasília, DF: MMA, 2016b.

Ministério do Meio Ambiente. Plano Nacional de Adaptação à Mudança do Clima: volume 2 - estratégias setoriais e temáticas. Brasília, DF: MMA, 2016c. n.1, 2016d.

Ministério do Meio Ambiente. Revista Adaptação à Mudança do Clima, v.1,

CAMPOS, I. et al. Understanding climate change policy and action in Portuguese municipalities: A survey. Land Use Policy, v.62, p.68-78, 2017.

CANEY S. Cosmopolitan justice, rights and global climate change. Canadian Journal of Law and Jurisprudence, p.255-78, 2005.

CARVALHO, A. Media (ted) discourses and climate change: a focus on political subjectivity and (dis) engagement. Wiley Interdisciplinary Reviews: Climate Change, v.1.2, p.172-9, 2010.

COLEN, J. Comentário ao texto 'nações, gerações e justiça climática', de Axel Gosseries. Revista Diacritica, v.28, n.2, p.333-41, 2014.

DALLA-NORA, G.; SATO, M. Pontes nas securas das águas: reflexões sobre as mudanças climáticas e justiça climática em comunidades quilombolas. Ciência Geográfica, Bauru, ano XXIII, v.XXIII, n.1, jan./dez., 2019.

DELICADO, A.; FIGUEIREDO, E.; SILVA, L. Community perceptions of renewable energies in Portugal: Impacts on environment, landscape and local development. Energy Research \& Social Science, v.13, p.84-93, 2016.

ESPINDOLA, I. B.; RIBEIRO, W. C. Cidades e mudanças climáticas: desafios para os planos diretores municipais brasileiros. Cad. Metrop., São Paulo, v.22, n.48, p.365-96, 2020.

FONSECA, P. M. O desafio do futuro no presente: a inconsistência temporal e a política das alterações climáticas. Relações Internacionais, p.137-150, 2014.

GOSSERIES, A. Nações, gerações e justiça climática. Revista Diacrítica, v.28, n.2, p.273-88, 2014.

GOUVEIA, J. P.; PALMA, P.; SIMÕES, S. G. Energy poverty vulnerability index: A multidimensional tool to identify hotspots for local action. Energy Reports, v.5, p.187$201,2019$.

GUERRA, J.; SCHMIDT L. Concretizar o wishful thinking - dos ODS à COP 21DOS ODS À COP21. Ambiente \&́ Sociedade, v.19, n.4, p.197-214, 2016.

HENRIQUE, K. P.; TSCHAKERT, P. Pathways to urban transformation: From dispossession to climate justice. Progress in Human Geography, October 2020. doi:10.1177/0309132520962856

HORTA, A. et al. Energy poverty in Portugal: Combining vulnerability mapping with household interviews. Energy and Buildings, n.203, 2019.

IORIS, A.; IRIGARAY, C.; GIRARD, P. Institutional responses to climate change: opportunities and barriers for adaptation in the Pantanal and the Upper Paraguay River Basin. Climatic Change, v.127, p.139-51, 2014. 
KASHWAN, P. Democracy in the Woods: Environemtnal Conservation and Social Justice in India, Tanzania, and Mexico. New York: Oxford University Press, 2017.

KASHWAN, P.; BIERMANN, F.; GUPTA, A.; C. OKEREKE. Planetary justice: Prioritizing the poor in earth system governance. Earth System Governance, Volume 6, December 2020, 100075.

KASHWAN, P. Climate Justice in the Global North: An Introduction. Case Studies in the Environment, p. 1-13, 2021.

MILANEZ, B.; FONSECA, I. F. Justiça climática e eventos climáticos extremos: o caso das enchentes no Brasil. Brasília: Ipea, 2010.

Justiça climática e eventos climáticos extremos: Uma análise da percepção social no Brasil. Revista Terceiro Incluido, v.1, n.2, p.82-100, 2011.

Climate justice: framing a new discourse in Brazil. Local Environment, v.17, n.10, p.1063-73, 2012.

MILLER, D. National Responsibility and Global Justice. Oxford: Oxford Press, 2007.

MIRANDA, A. C. de; FIRPO PORTO, M. Reflexões sobre a Rio + 20, a Cúpula dos Povos e a Saúde Coletiva. Saúde em Debate, v.36, p.68-76, 2012.

MMA. Secretaria de Mudanças Climáticas e Qualidade Ambiental. Subsídios ao processo de elaboração do Plano Nacional de Adaptação (PNA): Relatório da Consulta Pública 2014. MMA: Brasília, 2014.

Secretaria de Mudanças Climáticas e Qualidade Ambiental. Departamento de Licenciamento e Avaliação Ambiental. Relatório do Processo de Consulta Pública do Plano Nacional de Adaptação à Mudança do Clima. Brasília, 2015.

NUNES, A. R. The contribution of assets to adaptation to extreme temperatures among older adults. Plos One, v.13, 2018.

O'RIORDAN, T.; GOMES, C.; SCHMIDT, L. The difficulties of designing future coastlines in the face of climate change. Landscape Research, v.39.6, p.613-30, 2014.

OLIVEIRA, F. A nação e a arena internacional: desafios climáticos globais. ethic - $A n$ international Journal for Moral Phylosophy, v.16, n.1, p.83, 2017.

OTTO, D. et al. Can MOOCs Empower People to Critically Think about Climate Change? A Learning Outcome Based Comparison of Two MOOCs. Journal of Cleaner Production, v.222, p.12-21, 2019.

RAMMÊ, R. S. A política da justiça climática: conjugando riscos, vulnerabilidades e injustiças decorrentes das mudanças climáticas. Revista de Direito Ambiental, v.65, p.367, 2012 .

SANTOS, B. de S. The Future of the World Social Forum: The work of Translation. Development, v.48, p.15-22, 2005.

SAREEN, S.; HAARSTAD, H. Bridging socio-technical and justice aspects of sustainable energy transitions. Applied Energy, Elsevier, v.228(C), p.624-32, 2018.

SCHMIDT, L., DELICADO, A. Alterações climáticas na opinião pública. In: SCHMIDT, L.; DELICADO, A. (Ed.). Ambiente, alterações climáticas, alimentação e energia: a opinião dos portuguese. Lisboa: Imprensa de Ciências Sociais, 2014.

SCHMIDT, L. et al. Mudanças climáticas e económicas na costa portuguesa: percep- 
ções das comunidades, justiça social e democratização. In: VII CONGRESSO PORTUGUÊS DE SOCIOLOGIA, SOCIEDADE, CRISE E RECONFIGURAÇÕES, 2012. S. 1., 2012.

SCHMIDT, L. et al. Adapting Governance for Coastal Change in Portugal. Land Use Policy, p.314-25, 2013.

SCHMIDT, L. et al. Are we all on the same boat? The challenge of adaptation facing Portuguese coastal communities: Risk perception, trust-building and genuine participation. Land Use Policy, v.38, p.355-65, 2014.

SIMÕES, S. G.; GREGÓRIO, V.; SEIXAS, J. Mapping fuel poverty in Portugal. In: ENERGY ECONOMICS IBERIAN CONFERENCE, EEIC 2016. ELSEVIER SCIENCE BV, p.155-165, 2016.

TORRES, P. H. C. et al. Is the Brazilian National Climate Change Adaptation Plan Addressing Inequality? Climate and Environmental Justice in a Global South Perspective. Environ. Justice 2020, v.13, p.42-6, 2020.

TORRES, P. H. C. et al. Data and knowledge matters: Urban adaptation planning in São Paulo, Brazil. Urban Climate, v.36, 2021.

VERDAN, T. L. Injustiça climática? Um contemporâneo conceito em sede de injustiça ambiental. Conteúdo Juridico, Brasília, 2016.

Direitos humanos climáticos: a injustiça climática como potencializadora do alargamento dos Direitos Humanos. Conteúdo Jurídico, Brasilia, 2017.

VIEGAS, V. et al. Alterações Climáticas, Percepções e Racionalidades. Revista de Gestão Costeira Integrada, v.14, n.3, p.347-63, 2014.

ZAGONARI, F. Sustainable, Just, Equal, and Optimal Groundwater Management Strategies to Cope with Climate Change: Insights from Brazil. Water Resources Management, v.24, n. 13, p.3731-56, 2010.

RESUMO - Políticas, planos e estratégias de adaptação às mudanças climáticas têm ganhado agenda de governos em todo planeta e em diversas escalas. Estariam os atuais instrumentos endereçando a redução de desigualdades, justiça e demanda por direitos? A primeira parte da pesquisa analisa a produção científica no Brasil e em Portugal sobre justiça climática. A segunda parte discute como as estratégias e políticas atuais de adaptação nos dois países contêm componentes relacionados à justiça.

PALAVRAS-Chave: Justiça climática, Brasil, Portugal, Adaptação.

ABSTRACT - Policies, plans and strategies for adapting to climate change are now in the agenda of governments across the planet and at different scales. Are the current instruments addressing issues of justice, the reduction of inequality, and demands for rights? The first part of the research analyzes scientific production on climate justice in Brazil and Portugal. The second part discusses how current adaptation strategies and policies in both countries contain components related to justice.

KErwords: Climate justice, Brazil, Portugal, Adaptation 
Pedro Henrique Campello Torres é pesquisador do Instituto de Energia e Ambiente (IEE), Universidade de São Paulo. @ - phcampellotorres@gmail.com / https://orcid.org/0000-0002-0468-4329.

Alberto Matenhauer Urbinattié pós-doutorando no Departamento de Política Científica e Tecnológica, Universidade Estadual de Campinas.

@ - albertourbinatti@gmail.com / https://orcid.org/0000-0002-8257-0167.

Carla Gomes é pesquisadora do Instituto de Ciências Sociais (ICS) da Universidade de Lisboa. @ - carla.gomes@ics.ulisboa.pt / https://orcid.org/0000-0002-6089-0485.

Luisa Schmidt é professora do Instituto de Ciências Sociais (ICS) da Universidade de Lisboa. @-mlschmidt@ics.ulisboa.pt / https://orcid.org/0000-0002-7449-8636.

Ana Lia Leonel é doutoranda no Programa de Pós-Graduação em Planejamento e Gestão do Território (PGT), Universidade Federal do ABC (UFABC).

@ - analialeonel@gmail.com / https://orcid.org/0000-0003-4914-143X.

Sandra Momm é professora no Programa de Pós-Graduação em Planejamento e Gestão do Território (PGT), Universidade Federal do ABC (UFABC).

@ - sandra.momm@ufacb.edu.br / https://orcid.org/0000-0002-9724-5375.

Pedro Roberto Jacobi é professor titular senior no Programa de Pós-Graduação em Ciência Ambiental (Procam), Instituto de Energia e Ambiente (IEE), Universidade de São Paulo (USP). @ - prjacobi@gmail.com / http://orcid.org/0000-0001-6143-3019.

Recebido em 25.5.2020 e aceito em 18.2.2021.

I,VII Universidade de São Paulo, Instituto de Energia e Ambiente, São Paulo, Brasil.

II Universidade Estadual de Campinas, Departamento de Política Científica e Tecnológica, Campinas, São Paulo, Brasil.

III,IV Universidade de Lisboa, Instituto de Ciências Sociais, Lisboa, Portugal.

V,VI Universidade Federal do ABC, Programa de Pós-Graduação em Planejamento e Gestão do Território, Santo André, São Paulo, Brasil 